\title{
Vibration Response and Stress Analysis of Planar Elastic Tube Bundle Induced by Fluid Flow
}

\author{
De-Rong Duan ${ }^{1}$, Pei-Qi Ge ${ }^{1,2^{*}}$, Wen-Bo Bi ${ }^{1}$ and Yan-Ying Dong ${ }^{1}$
}

\begin{abstract}
Flow-induced vibration plays a positive role on heat transfer enhancement. Meanwhile, it is also a negative factor for fatigue strength. Satisfying the fatigue strength is the primary prerequisite for heat transfer enhancement. This paper numerically studied the flow-induced vibration of planar elastic tube bundle based on a two-way fluid-structure interaction (FSI) calculation. The numerical calculation involved the unsteady, three-dimensional incompressible governing equations solved with finite volume approach and the dynamic balance equation of planar elastic tube bundle solved with finite element method combined with dynamic mesh scheme. The numerical approach was verified by comparing with the published experimental results. Then the vibration trajectory, deformation and stress contour of planar elastic tube bundle were all studied. Results show that the combined movement of planar elastic tube bundle represents the agitation from inside to outside. The vibration of out-of-plane is the main vibration form with the typically sinusoidal behavior because the magnitude of displacement along the out-of-plane direction is the 100 times than the value of in-plane direction. The dangerous point locates in the innermost tube where the equivalent stress can be utilized to study the multiaxial fatigue of planar elastic tube bundle due to the alternating stress concentration. In the velocity range of $0.2-3 \mathrm{~m} / \mathrm{s}$, it is inferred that the vibration amplitude plays a role on the stress response and the stress amplitude is susceptible to the fluid velocity. This research paves a way for studying the fatigue strength of planar elastic tube bundle by flow-induced vibration.
\end{abstract}

Keywords: Flow-induced vibration, Planar elastic tube bundle, Vibration response, Stress analysis

\section{Introduction}

Flow-induced vibration, considered as a complex phenomenon of fluid-structure interaction, may occur in many engineering fields, such as heat exchanger, nuclear engineering, ocean engineering [1-3]. This vibration will lead to the fatigue of structure due to the periodic alternating force. Thus, many scholars pay their attention on the fatigue failure by flow-induced vibration.

Ji et al. [4] analyzed the flow-induced vibration of heat transfer tube in evaporator heating chamber. The shedding frequency of vortex street, natural frequency and critical flow velocity were all calculated. Results show

\footnotetext{
*Correspondence: pqge@sdu.edu.cn

1 School of Mechanical Engineering, Shandong University, Jinan 250061, China

Full list of author information is available at the end of the article
}

that the Karman vortex and turbulence buffeting occur at the entrance with high velocity of water vapor. While, the resonance of heat transfer tube occur because the shedding frequency is similar to the natural frequency of tube. The coupling effect of vibration and temperature difference stress lead to the fatigue damage of tube. In ocean engineering, fatigue failure of marine risers due to flowinduced vibration is one of the most significant failure modes. Xue et al. [5] proposed a new prediction model of FIV fatigue damage for riser considering both crossflow and in-line vibrations based on the energy equilibrium theory. The effect of top velocity of sheared current, top tension, fluid density and the material of structure on the fatigue damage were discussed. Results reveal that the displacement of in-line vibration is much less than the value of cross-flow. However, there is a same important level for both fluid flows because high vibration
Springer Open

(c) The Author(s) 2018. This article is distributed under the terms of the Creative Commons Attribution 4.0 International License (http://creativecommons.org/licenses/by/4.0/), which permits unrestricted use, distribution, and reproduction in any medium, provided you give appropriate credit to the original author(s) and the source, provide a link to the Creative Commons license, and indicate if changes were made. 
frequency occurs in in-line vibration. The top velocity of sheared current plays a great role on the fatigue damage. The fatigue damage may be reduced $50 \%$ owing to the $10 \%$ reduction in top velocity of sheared current. Sun et al. [6] investigated the fatigue damage of riser undergoing in-line and cross-flow flow-induced vibration using pseudo-excitation method. Results show that the position near the top of the riser $\mathrm{Z} / \mathrm{LR}=0.9$ is the dangerous point. The fatigue damage is mainly subjected to the cross-flow vibration. Wang et al. [7] experimentally studied the fatigue damage caused by vortex-induced vibration based on rain-flow counting and a standard $S-N$ curve in an ocean basin. Results show that amplitude modulation and mode transition dominate the fatigue of flexible cylinder. Ei et al. [8] studied the effect of tube support clearance on the flow-induced vibration of steam generators. Special emphasis was placed on crack propagation and leakage rates. The developed model was established based on the stress-time history due to the flow-induced vibration. Results show that clearances below $0.3 \mathrm{~mm}$ are crucial to restricting crack growth rates to acceptable levels.

Every coin has two sides. This vibration is a negative factor for fatigue failure. However, Flow-induced vibration has been proved to be a positive role on heat transfer enhancement [9-11]. Planar elastic tube bundle is a novel heat transfer device to enhance heat transfer using flow-induced vibration [12]. The heat transfer may be enhanced by two times than the value of fixed tube bundle [13]. Some emphasis has been placed on the heat transfer characteristics and the vibration characteristics [14-16]. For the fatigue failure of planar elastic tube bundle, there is very less researches in the open literatures. Yan et al. [17] pointed out that the cross section diameter of tube bundle and the mass block played major role on the stress of tube bundle.

Satisfying the fatigue strength is the primary prerequisite for heat transfer enhancement. In order to enrich the study of planar elastic tube bundle, the vibration response and stress analysis are studied in this paper to pave the way for fatigue analysis of planar elastic tube bundle. Thus, a two-way fluid-structure interaction is conducted for the flow-induced vibration of planar elastic tube bundle. The vibration trajectory, deformation and stress contour of planar elastic tube bundle are all studied. The conclusions are the basis for investigating the fatigue failure of planar elastic tube bundle.

\section{Numerical Approach}

\subsection{Physical Model}

The schematic of heat exchanger with single-row planar elastic tube bundle presented in this paper is shown in Figure 1(a). The height $H_{s}$ and diameter $D_{s}$ of heat

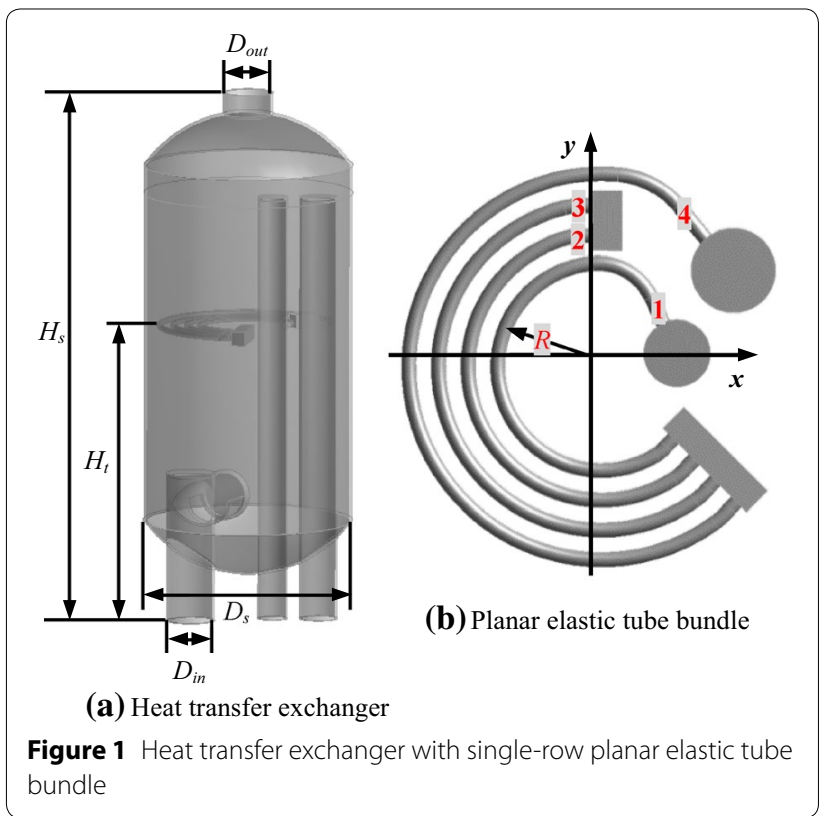

exchanger are $780 \mathrm{~mm}$ and $300 \mathrm{~mm}$, respectively. The diameter of inlet $D_{\text {in }}$ and outlet $D_{\text {out }}$ are $63 \mathrm{~mm}$ and $65 \mathrm{~mm}$, respectively. The planar elastic tube bundle is installed in the middle of shell-side. The installation height $H_{t}$ is $430 \mathrm{~mm}$. The planar elastic tube bundle is shown in Figure 1(b) including four curved copper tube and two mass-blocks. The radius of innermost tube $R_{1}$ is $70 \mathrm{~mm}$ and the tube spacing is $20 \mathrm{~mm}$. In the present work, the diameter of tube is $10 \mathrm{~mm}$ and the tube wall thickness is $1.5 \mathrm{~mm}$.

In the heat transfer process, the fluid of shell-side flows into heat exchanger from the bottom inlet and the planar elastic tube bundle is heated by the fluid of tube-side. Then the fluid of shell-side is heated and flows out from the top outlet. Meanwhile, the tube vibration occurs because of the periodic alternating force. It is verified that this vibration is beneficial to heat transfer [18-20]. In this paper, the flow-induced vibration by shell-side is studied because the effect of tube-side on the vibration of planar elastic tube bundle can be ignored [21].

\subsection{Governing Equations}

As described above, a so-called two-way FSI is indispensable to study the flow-induced vibration of planar elastic tube bundle. In the present work, the fluid of shell-side is considered as three-dimensional, unsteady and incompressible water. Therefore, in the Arbitrary Lagrangian-Eulerian (ALE) frame [22-24], this is used to calculate the two-way FSI approach. The governing equations including continuity, momentum and energy equation for the shell-side fluid domain are listed as follows: 


$$
\begin{aligned}
& \nabla\left(u_{f}-u_{m f}\right)=0, \\
& \frac{\partial u_{f}}{\partial t}+\left(u_{f}-u_{m f}\right) \nabla u_{f}=-\frac{\nabla P}{\rho}+v \nabla^{2} u_{f}, \\
& \rho C_{p} \frac{\partial T}{\partial t}+\rho C_{p}\left(u_{f}-u_{m f}\right) \nabla T=\lambda \nabla^{2} T,
\end{aligned}
$$

where $\left(u_{f}-u_{m f}\right)$ is the convective velocity in shell-side domain. $u_{f}$ is the fluid velocity and $u_{m f}$ is the mesh velocity. $T, p, \rho, v, C_{p}$ and $\lambda$ represent the temperature, pressure, density, kinematic viscosity, specific heat and thermal conductivity of fluid.

In the present work, the commercial CFD code ANSYS CFX and ANSYS Transient Structural Module were utilized to solve the flow-induced vibration of planar elastic tube bundle. Figure 2 shows the main flowchart of flow-induced vibration calculation in this paper. The fluid flow in shell-side calculated by CFX is conducted firstly because the tube vibration occurs due to the periodic alternating force. The displacement of planar elastic tube bundle is transferred to the domain of shell-side. As a result, the boundary of planar elastic tube bundle in shell-side is updated using moving mesh method [25, 26]. After the CFD analysis, the force generated on planar elastic tube bundle is transferred to the domain of structure. Then, the structural motion equation of planar

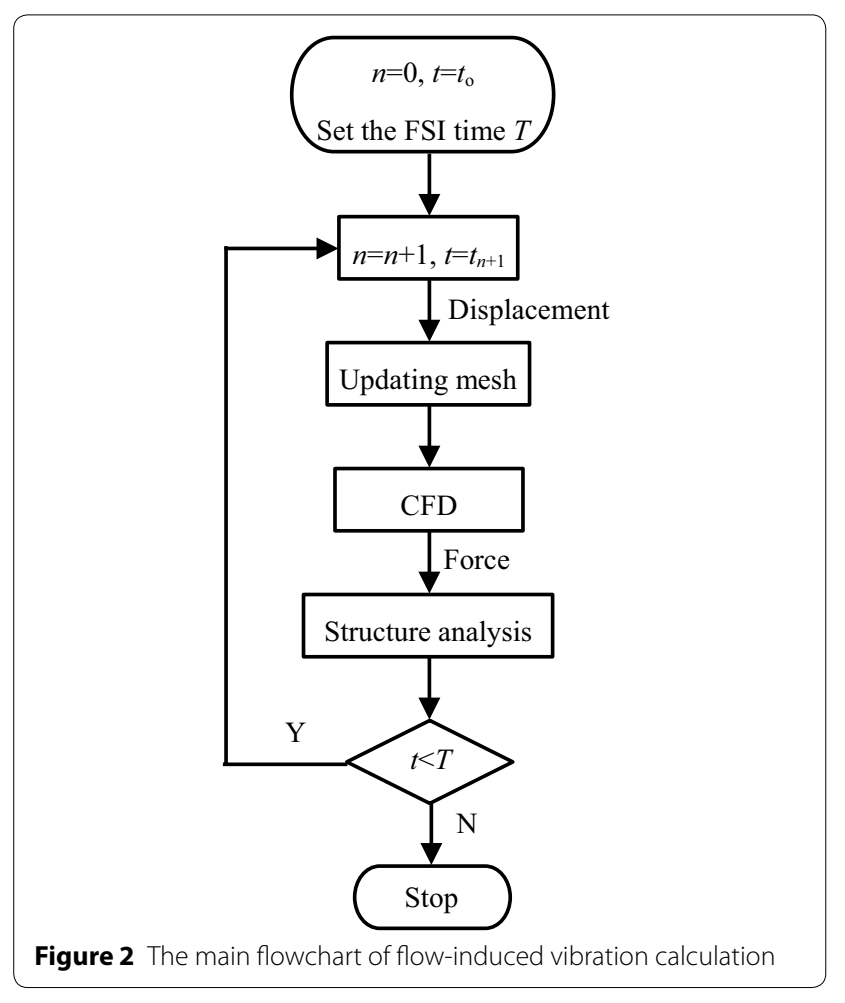

elastic tube bundle is calculated based on the above force boundary. Consequently, the flow-induced vibration of planar elastic tube bundle in shell-side is calculated by this sequential solution of bi-direction fluid-structure coupling method.

Owing to the two-way FSI coupling calculation, the displacement and force are transferred on the fluidstructure interface [27]. Thus, the following conservation equations must be satisfied on the fluid-structure interface:

$$
\begin{aligned}
& d_{f}=d_{s}, \\
& n \cdot \sigma_{f}=n \cdot \sigma_{s},
\end{aligned}
$$

where $d$ and $\sigma$ represent the displacement and stress on the fluid-structure interface. $n$ is the normal vector.

\subsection{Meshing and Boundary Conditions}

Figure 3 shows the mesh of shell-side and structure domain. In Figure 3(a), the shell-side was divided into three sections including outlet section, middle section and inlet section due to the complex nature of shell-side geometry structure. Among which, the outlet section and middle section were discretized by hexahedral mesh to save the computing memory and time. Meanwhile, the boundary layers with the first spacing $1 \times 10^{-4}$ and growth ratio 1.2 were established around the planar elastic tube bundle. The inlet section was discretized by tetrahedral mesh. At last, three sections were connected by Interface 1 and Interface 2 . The inlet was set to velocityinlet boundary with the constant temperature. The outlet was set to pressure boundary condition with 0 (gauge). The wall of planar elastic tube bundle was set to no-slip

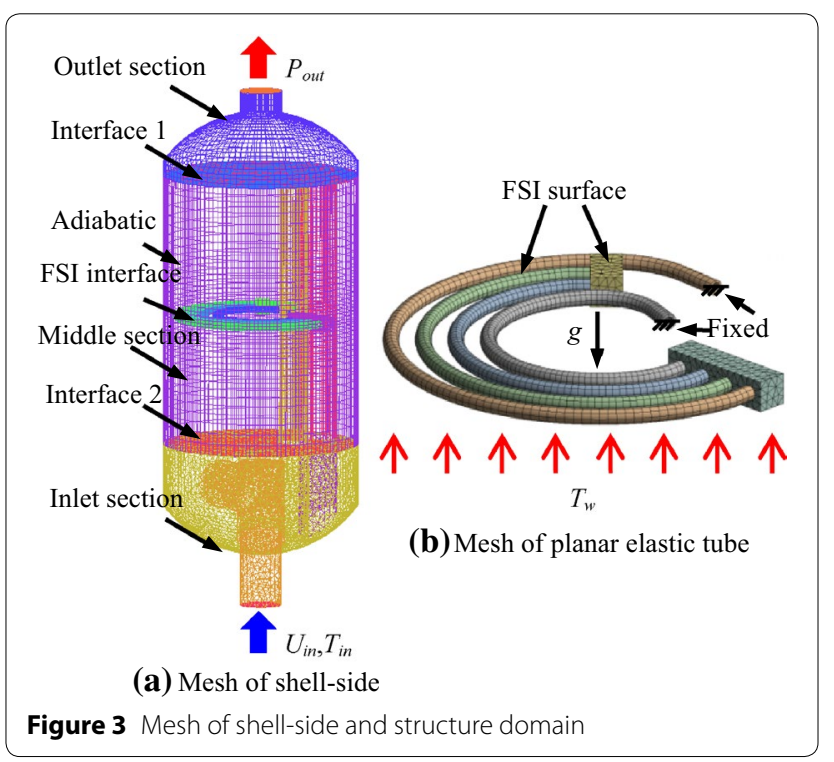


boundary condition with constant wall temperature and all surface of planar elastic tube bundle were set to FSI interface for data transfer. Others wall of shell-side were set to adiabatic. In Figure 3(b), the four curved were discretized by hexahedral mesh and two mass-blocks were discretized by tetrahedral mesh. The innermost and outermost tubes were fixed. All of the surfaces of planar elastic tube bundle were set to FSI surface with the constant wall temperature. The earth gravity is applied along the $z$ direction.

\subsection{Validation and Grid Independence Test}

In order to solve the two-way FSI accurately, the comparison between numerical results and experimental results were conducted to validate the accuracy of flow-induced vibration calculation. In Table 1, it is found that a litthe relative error occurs between numerical results and experimental results. The smallest error and the greatest error are $1.32 \%$ and $2.41 \%$, respectively. It is validated that the numerical results are acceptable for the flowinduced vibration calculation.

Meanwhile, three kinds of grid number in shell-side were established to conduct the grid independence test, 316723,323123 and 329523 . It is derived that the relative error of convective heat transfer coefficient between 316723 and 323123 is $0.23 \%$ and the relative error of convective heat transfer coefficient between 323123 and 329523 is $0.16 \%$. Almost equal vibration frequency of planar elastic tube bundle occurs for these three kinds of grid number. Therefore, the shell-side was discretized to 323123 grids to solve the flow-induced vibration calculation in view of saving computer resource and keeping a balance between computational economics and accuracy.

\section{Results and Discussion}

\subsection{Vibration Trajectory}

At present, it is not clear for the movement trajectory of planar elastic tube bundle. Owing to the numerical calculation in flow-induced vibration, the vibration trajectory of planar elastic tube bundle on three planes is shown in Figure 4. The red arrow represents the movement direction of planar elastic tube bundle. The clockwise movement occurs on the $x-y$ and $x-z$ plane. The counterclockwise movement occurs on the $y-z$ plane. It means that the combined movement of planar elastic tube bundle is the agitation from inside to outside, which is beneficial to reducing the thickness of thermal boundary layer to enhance heat transfer [29].

It is found that there is the same magnitude of displacement in $x$ and $y$ direction. The magnitude of displacement in $z$ direction is 100 times than the value in $x$ and $y$ direction. It means that the vibration direction is perpendicular to the horizontal plane of planar elastic tube bundle, which is agreed with the conclusion of out-of-plane vibration [15].

Stable vibration is the premise to study the flowinduced vibration of planar elastic tube bundle. Thus, the time history of vibration in planar elastic tube bundle is shown in Figure 5 when the inlet velocity is in the range of $0.2-0.5 \mathrm{~m} / \mathrm{s}$. It is clear that the stable vibration is obtained after $0.76 \mathrm{~s}$ in calculation of two-way FSI. The vibration curve represents typically sinusoidal behavior. For the vibration amplitude, it is derived that the magnitude of displacement belongs to the sub-millimeter level. The vibration amplitude increases with the increase in inlet velocity due to the increasing fluid force. While, there is almost equal frequency $24.28 \mathrm{~Hz}$ in these inlet velocities owing to the step evolution characteristic in vibration frequency of planar elastic tube bundle [21].

\subsection{Deformation}

When the stable vibration is obtained, the deformation of planar elastic tube bundle subjected to flow-induced vibration in a period is shown in Figure 6. For the vibration amplitude, it is magnified by 100 times to show clearly because of the vibration amplitude within the submillimeter level.

It is found that that the direction of vibration is along the $z$ direction. The vibration balance position is below the horizontal plane of planar elastic tube bundle subjected to the earth gravity. The red arrow represents the instantaneous vibration direction. At the time of $\tau / 4$ and $3 \tau / 4$, the planar elastic tube bundle reaches the position of peak and valley, respectively. For the velocity $0.2 \mathrm{~m} / \mathrm{s}$,

Table 1 Validation of the calculation in flow-induced vibration

\begin{tabular}{lllll}
\hline Inlet velocity $(\mathbf{m} / \mathbf{s})$ & Monitoring points & \multicolumn{2}{l}{ Vibration frequency $f(\mathbf{H z})$} & Relative error $(\%)$ \\
\cline { 3 - 4 } & & Numerical results & Experimental results [28] & 2.04 \\
\hline 0.2 & Big MB & 28.57 & 28.0 & 2.41 \\
0.4 & Small MB & 29.7 & 29.0 & 1.32 \\
& Big MB & 28.37 & 28.0 & 2.17 \\
\hline
\end{tabular}



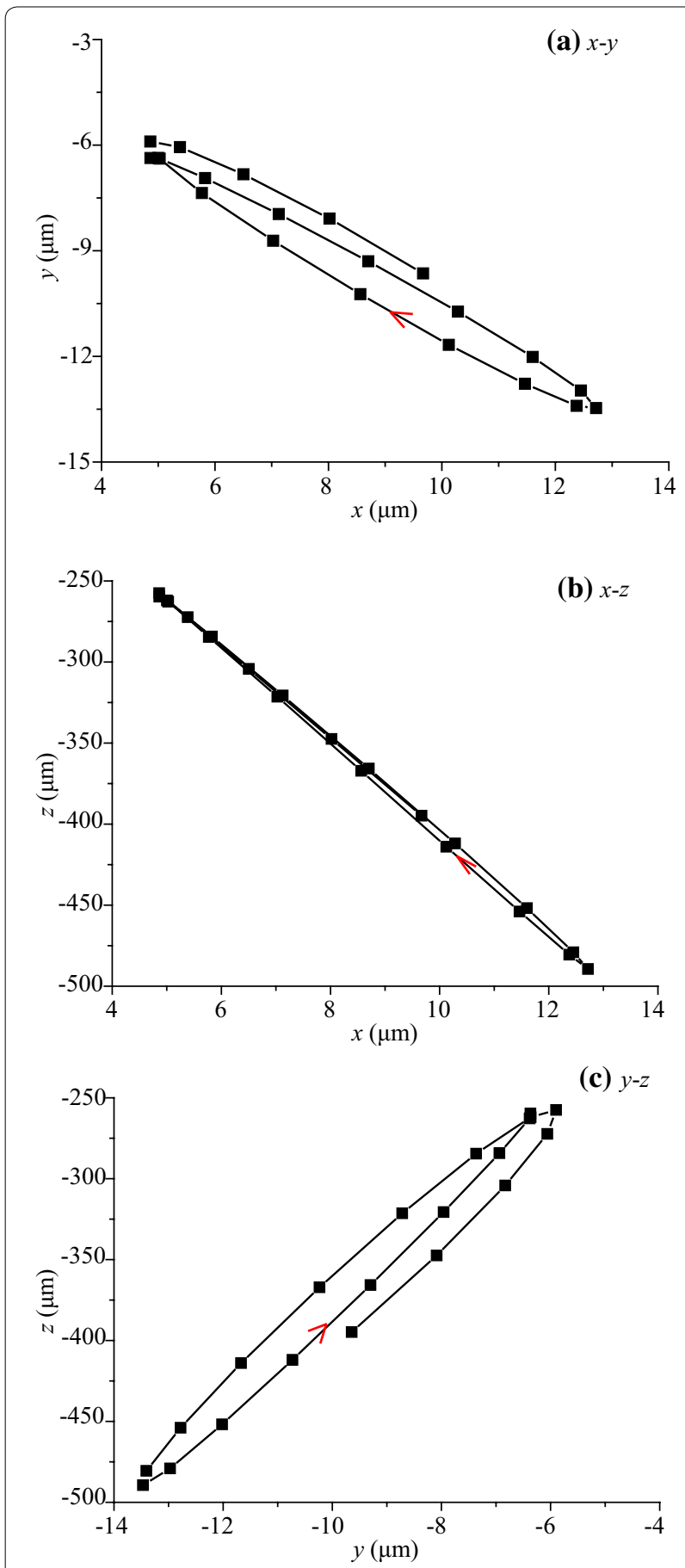

Figure 4 The vibration trajectory of planar elastic tube bundle

the planar elastic tube bundle is still below the horizontal plane at the position of peak. While the planar elastic tube bundle is almost over the horizontal plane at the position of peak in the velocity $0.5 \mathrm{~m} / \mathrm{s}$. The reason behind this is that the fluid force generated by increasing velocity overcomes the effect of earth gravity. It means

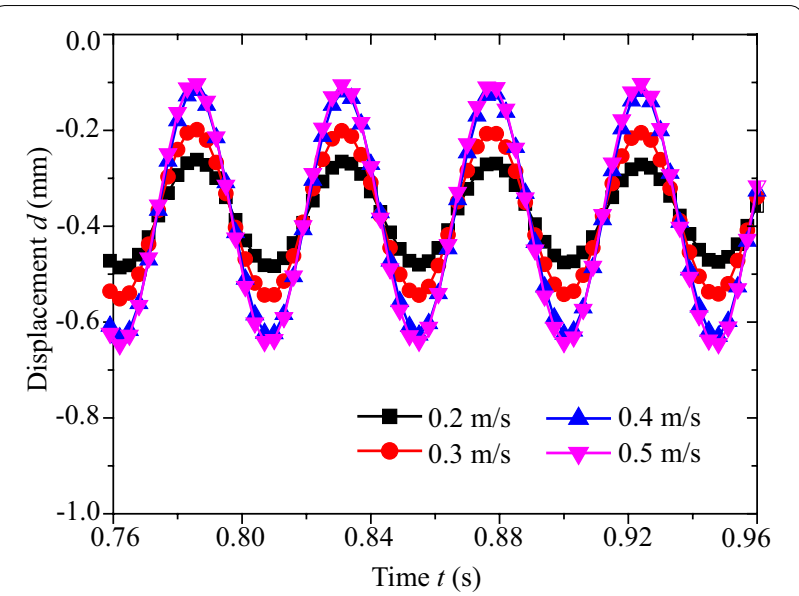

Figure 5 Time history of vibration in planar elastic tube bundle

that the vibration balance position will be raised because of the increasing fluid force.

\subsection{Stress Analysis}

Stress concentration of planar elastic tube bundle will occur in the vibration process as shown in Figure 6. Usually, the dangerous point locates in the innermost tube because the innermost tube is fixed with the greatest curvature. In Figure 6, it is clear that the maximum stress occurs at the time of $3 \tau / 4$, where is the valley of vibration. The value of stress at dangerous point is various with the vibration of planar elastic tube bundle. As a result, the fatigue failure will occur due to the alternating stress concentration in planar elastic tube bundle. It is verified that the planar elastic tube bundle suffers from bending stress and torsional stress. Thus, the equivalent stress can be utilized to study the multiaxial fatigue of planar elastic tube bundle, which is established based on the Von Mises stress criteria [30].

Figure 7 shows the time history of stress at dangerous point in the velocity $0.2 \mathrm{~m} / \mathrm{s}$ and $0.5 \mathrm{~m} / \mathrm{s}$. It is clear that the stress curve represents typically sinusoidal behavior, which is similar to the vibration curve showed in Figure 5. The stress amplitude increases with the increases in the velocity. It is derived that the stress amplitude is $2.0 \mathrm{MPa}$ and $4.5 \mathrm{MPa}$ in the velocity $0.2 \mathrm{~m} / \mathrm{s}$ and $0.5 \mathrm{~m} / \mathrm{s}$, respectively. Meanwhile, almost equal mean stress 7.1 $\mathrm{MPa}$ occurs in these two velocities. It means that the stress amplitude is sensitive to the fluid flow in the velocity range of $0.2-0.5 \mathrm{~m} / \mathrm{s}$.

\subsection{Vibration Response in High Velocity}

It is derived that the velocity of range for this single-row planar elastic tube bundle heat exchanger is $0.2-3 \mathrm{~m} / \mathrm{s}$ based on the velocity of range in the heat exchanger 


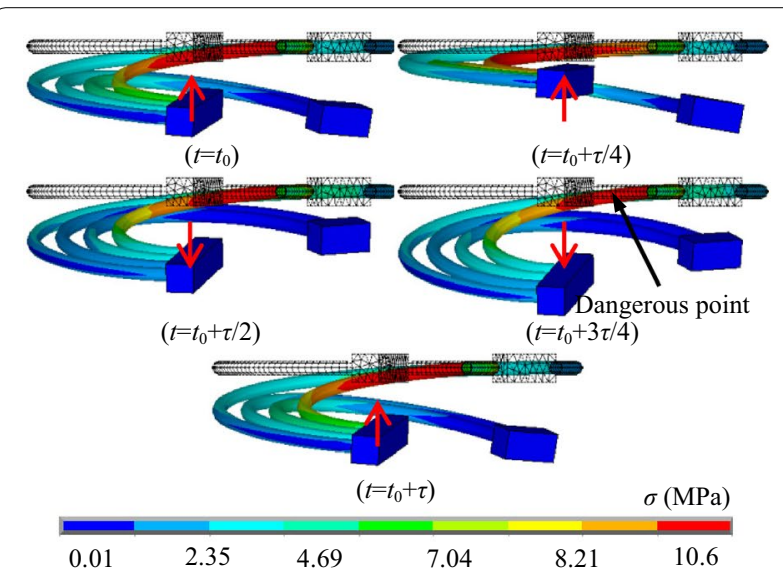

(a) $U_{\text {in }}=0.2 \mathrm{~m} / \mathrm{s}$

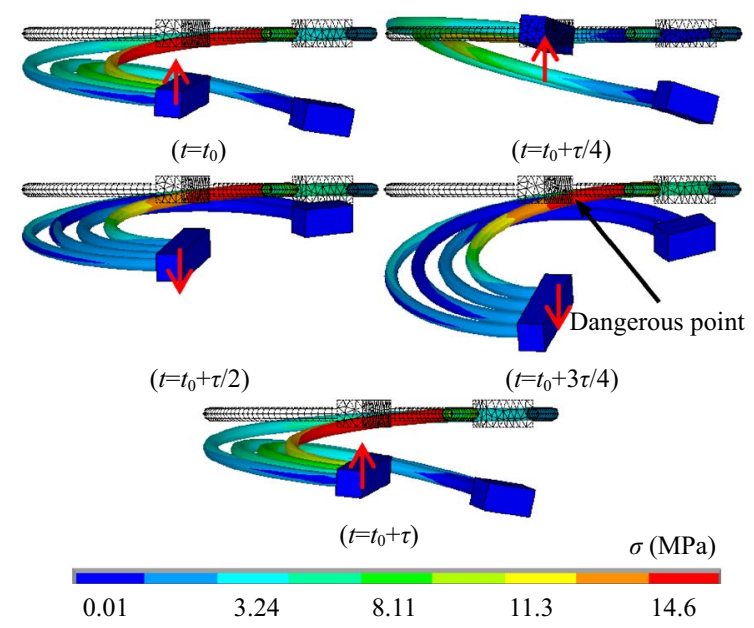

(b) $U_{i n}=0.5 \mathrm{~m} / \mathrm{s}$

Figure 6 Deformation and stress contour of planar elastic tube bundle

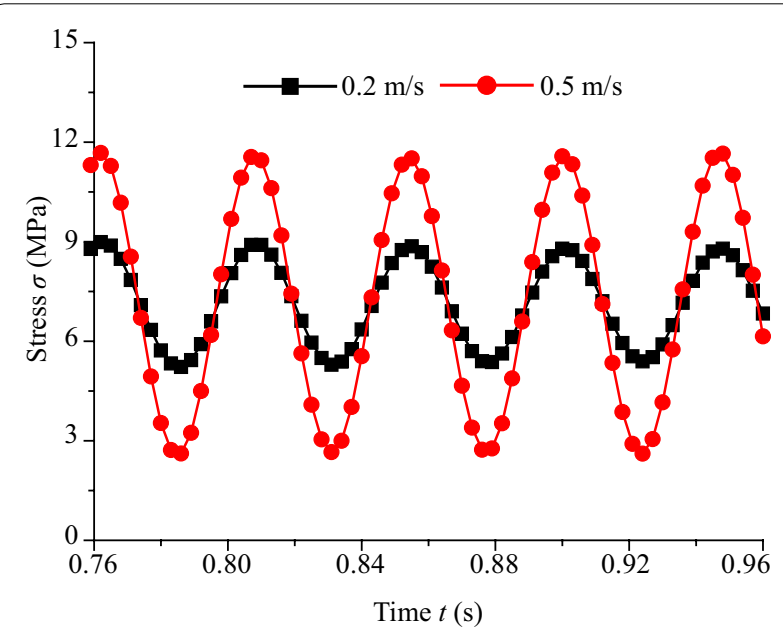

Figure 7 Time history of stress at dangerous point
SIW4.2/5-95/70-65/10. The vibration and stress response were studied in the low flow velocity range $0.2-0.5 \mathrm{~m} / \mathrm{s}$. Figure 8 shows the vibration and stress response of planar elastic tube bundle in the high velocity range of $1-3 \mathrm{~m} / \mathrm{s}$.

In Figure 8(a), the maximum vibration amplitude $0.48 \mathrm{~mm}$ occurs in the velocity $2 \mathrm{~m} / \mathrm{s}$, which is the 5.3 times than the value in the velocity $0.2 \mathrm{~m} / \mathrm{s}$. The vibration balance position is over the horizontal plane when the value of velocity exceeds $2 \mathrm{~m} / \mathrm{s}$. Then, the vibration amplitude decreases with the increases in the velocity when the velocity exceeds $2 \mathrm{~m} / \mathrm{s}$. The minimum vibration amplitude $0.4 \mathrm{~mm}$ is obtained in the velocity $3 \mathrm{~m} / \mathrm{s}$, which is the 4.4 times than the value in the velocity $0.2 \mathrm{~m} / \mathrm{s}$. It is derived that the fluctuation $20 \%$ occurs for the vibration amplitude in the velocity range of $1-3 \mathrm{~m} / \mathrm{s}$. For the vibration frequency, the maximum vibration frequency $23.69 \mathrm{~Hz}$ occurs in the velocity $2.5 \mathrm{~m} / \mathrm{s}$ and the minimum vibration frequency $23.33 \mathrm{~Hz}$ occurs in the velocity $1 \mathrm{~m} / \mathrm{s}$. Comprehensive analysis are conducted that the fluctuation $2 \%$ occurs for the vibration

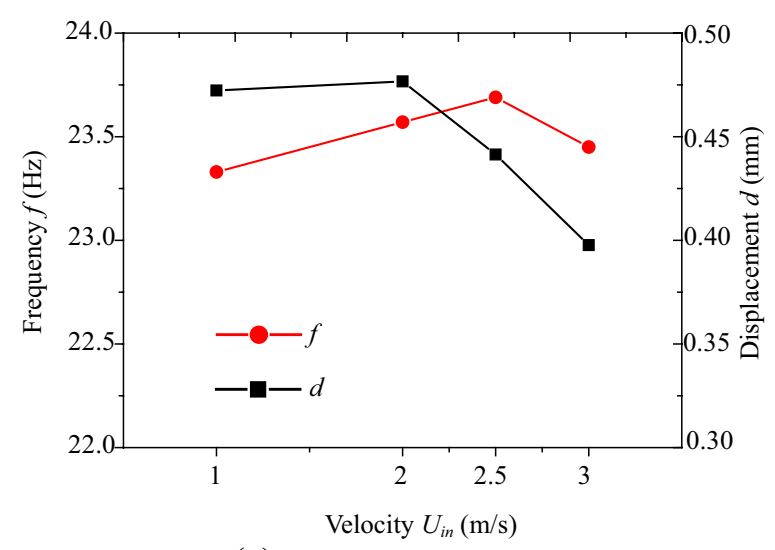

(a) Frequency and displacement

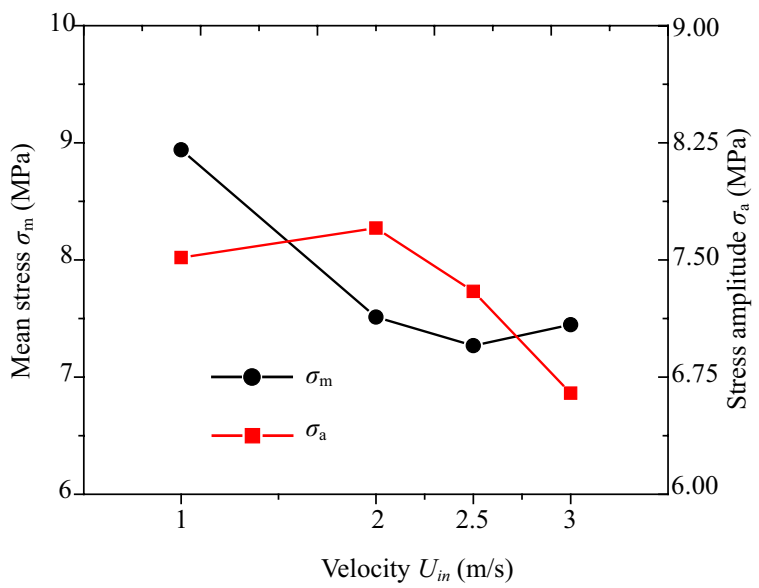

(b) Equivalent stress

Figure 8 Vibration and stress response in high velocity 
frequency in the velocity range of $1-3 \mathrm{~m} / \mathrm{s}$. It means that the vibration frequency exhibits independence on the inlet velocity. As a result, the vibration amplitude is more susceptible to the fluid velocity compared to the vibration frequency.

In Figure 8(b), the stress response is changed with the various in the vibration balance position and vibration amplitude. As a result, the mean stress decreases first and then increases. The maximum mean stress $9.0 \mathrm{MPa}$ occurs in the velocity $1 \mathrm{~m} / \mathrm{s}$. The stress amplitude increases first and then decreases. The maximum stress amplitude $7.7 \mathrm{MPa}$ occurs in the velocity $2 \mathrm{~m} / \mathrm{s}$. There is a larger fluctuation in stress amplitude compared the value in the velocity range $0.2-0.5 \mathrm{~m} / \mathrm{s}$. Comprehensive analysis is conducted for the vibration and stress response. It implies that the vibration amplitude plays a role on the stress response and the stress amplitude is susceptible to the fluid velocity.

\section{Conclusions}

A so-called two-way FSI is conducted to study the flowinduced vibration of planar elastic tube bundle based on finite volume approach and finite element method. The numerical calculation is verified by comparing with the experimental results. Vibration trajectory, deformation and stress analysis of planar elastic tube bundle are all investigated in the velocity $0.2-3 \mathrm{~m} / \mathrm{s}$. Some results of the present work are listed in the following:

(1) The combined movement of planar elastic tube bundle represents the agitation from inside to outside. The magnitude of displacement along the outof-plane direction is the 100 times than the value of in-plane direction, which means that the vibration of out-of-plane is the main vibration form of planar elastic tube bundle.

(2) The vibration of planar elastic tube bundle represents typically sinusoidal behavior. The vibration balance position is lifted with the increases in fluid force due to the increasing fluid velocity. Compared to the vibration frequency, the vibration amplitude is more susceptible to the fluid velocity.

(3) The dangerous point locates in the innermost tube because the innermost tube is fixed with the greatest curvature. The equivalent stress can be utilized to study the multiaxial fatigue of planar elastic tube bundle due to the alternating stress concentration.

(4) Comprehensive analysis is conducted for the vibration and stress response in the velocity range of $0.2-3 \mathrm{~m} / \mathrm{s}$. It is inferred that the vibration amplitude plays a role on the stress response and the stress amplitude is susceptible to the fluid velocity.
Authors' Contributions

P-QG was in charge of the whole trial; D-RD wrote the manuscript; W-BB and Y-YD assisted with sampling and laboratory analyses. All authors read and approved the final manuscript.

\section{Author details}

${ }^{1}$ School of Mechanical Engineering, Shandong University, Jinan 250061,

China. ${ }^{2}$ Key Laboratory of High Efficiency and Clean Mechanical Manufacture of Ministry of Education, Shandong University, Jinan 250061, China.

\section{Authors' Information}

De-Rong Duan, born in 1988, is currently a PhD candidate at School of Mechanical Engineering, Shandong University, China. He received his bachelor degree from University of Jinan, China, in 2010. His research interests include flow-induced vibration. Tel: +86-531-88399245; E-mail: dr_duan@126.com.

Pei-Qi Ge, born in 1963, is currently a professor at Shandong University, China. He received his PhD degree from Harbin Institute of Technology, China, in 1997. His research interests include flow-induced vibration, crystal slicing processing and diamond wire saw. Tel: +86-531-88399277; E-mail: pqge@sdu. edu.cn.

Wen-Bo Bi, born in 1979, is currently a lecturer at School of Mechanical Engineering, Shandong University, China. He received his PhD degree from Shandong University, China, in 2015. His main research interests include mechachonics engineering, crystal slicing processing and diamond wire saw. Tel: +86-531-88399277; E-mail: biwenbo@sdu.edu.cn.

Yan-Ying Dong, born in 1991, is currently a master candidate at School of Mechanical Engineering, Shandong University, China. She received her bachelor degree from Harbin University of Commerce, China, in 2015. Her research interests include flow-induced vibration. Tel: +86-531-88399245; E-mail: dongyanying7@163.com.

Competing Interests

The authors declare that they have no competing interests.

Ethics Approval and Consent to Participate

Not applicable.

Funding

Supported by National Natural Science Foundation of China (Grant No. 51475268), National Basic Research Program of China (973 Program, Grant No. 2007CB206903)

\section{Publisher's Note}

Springer Nature remains neutral with regard to jurisdictional claims in published maps and institutional affiliations.

Received: 25 June 2017 Accepted: 16 April 2018

Published online: 26 April 2018

\section{References}

[1] J Yang, S Yuan, J Yuan, et al. Numerical and experimental study on flowinduced noise at blade-passing frequency in centrifugal pumps. Chinese Journal of Mechanical Engineering, 2014, 27(3): 606-614.

[2] K Yan, N Wang, Q Zhai, et al. Theoretical and experimental investigation on the thermal characteristics of double-row tapered roller bearings of high speed locomotive. International Journal of Heat and Mass Transfer, 2015, 84: 1119-1130.

[3] $\mathrm{C} \mathrm{Hu}, \mathrm{G}$ Wang, B Huang. Experimental investigation on cavitating flow shedding over an axisymmetric blunt body. Chinese Journal of Mechanical Engineering, 2015, 28(2): 387-393.

[4] W Ji, J M Fan, et al. Analysis on induced vibration failure of heat transfer tube in heating room of evaporator. Chemical Engineering \& Equipment, 2012(4): 22-24.

[5] H Xue, W Tang, X Qu. Prediction and analysis of fatigue damage due to cross-flow and in-line VIV for marine risers in non-uniform current. Ocean Engineering, 2014, 83: 52-62. 
[6] L Sun, C F Liu, Z Zong, et al. Fatigue damage analysis of the deepwater riser from VIV using pseudo-excitation method. Marine Structures, 2014, 37: $86-110$.

[7] J Wang, S Fu, R Baarholm, et al. Fatigue damage induced by vortexinduced vibrations in oscillatory flow. Marine Structures, 2015, 40: 73-91.

[8] B S El, M Hassan, J Riznic. A comprehensive flow-induced vibration model to predict crack growth and leakage potential in steam generator tubes. Nuclear Engineering and Design, 2015, 292: 17-31.

[9] L Yang, M Zhou, ZTian. Heat transfer enhancement with mixing vane spacers using the field synergy principle. Chinese Journal of Mechanical Engineering, 2017, 30(1): 127-134.

[10] K Yan, Y Wang, Y Zhu, et al. Investigation on heat dissipation characteristic of ball bearing cage and inside cavity at ultra high rotation speed. Tribology International, 2016, 93: 470-481.

[11] A Raisi, A Qanbary. Effect of magnetic field on forced convection between two nanofluid laminar flows in a channel. Chinese Journal of Mechanical Engineering, 2016, 29(6): 1235-1243.

[12] J D Ji, P Q Ge, W B Bi. Numerical analysis on shell-side flow-induced vibration and heat transfer characteristics of elastic tube bundle in heat exchanger. Applied Thermal Engineering, 2016, 107: 544-551.

[13] D R Duan, P Q Ge, W B Bi, et al. Numerical investigation on synthetical performance of heat transfer of planar elastic tube bundle heat exchanger. Applied Thermal Engineering, 2016, 109: 295-303.

[14] D R Duan, P Q Ge, W B Bi. Numerical investigation of heat transfer characteristic of fixed planar elastic tube bundles. Energy Conversion and Management, 2015, 103: 859-870.

[15] B Jiang, M C Tian, W Hao, et al. Experiment and numerical simulation on inherent vibration characteristics of the new elastic tube bundle. Journal of Shandong University (Engineering Science), 2012, 4: 136-140, 146. (in Chinese)

[16] D R Duan, P Q Ge, W B Bi. Numerical investigation on heat transfer performance of planar elastic tube bundle by flow-induced vibration in heat exchanger. International Journal of Heat and Mass Transfer, 2016, 103: 868-878.

[17] K Yan, P Q Ge, Q Zhai. A comprehensive comparison on vibration and heat transfer of two elastic heat transfer tube bundles. Journal of Central South University, 2015, 22(1): 377-385.

[18] Y Su, M Li, M Liu, et al. A study of the enhanced heat transfer of flowinduced vibration of a new type of heat transfer tube bundle-The planar bending elastic tube bundle. Nuclear Engineering and Design, 2016, 309: 294-302.
[19] F Sastre, A Velazquez. Experimental study on heat transfer enhancement caused by the flow-induced vibration of a prism inside a channel. Applied Thermal Engineering, 2017, 125: 412-424.

[20] Y Zhu, F Bian, Y Wang, et al. Conjugate heat transfer analysis of an ultrasonic molten metal treatment system. Chinese Journal of Mechanical Engineering, 2014, 27(5): 986-991.

[21] J D Ji, P Q Ge, W B Bi. Numerical analysis on the combined flow induced vibration response of elastic tube bundle in heat exchanger. Journal of Xi'an Jiaotong Univeristy, 2015, 49(9): 24-29. (in Chinese)

[22] N G De, A Kalmbach, M Breuer, et al. Flow past a cylinder with a flexible splitter plate: A complementary experimental-numerical investigation and a new FSI test case (FSI-PfS-1a). Computers \& Fluids, 2014, 99: 18-43.

[23] Q Zhu, Y L Xu, L D Zhu, et al. Vortex-induced vibration analysis of longspan bridges with twin-box decks under non-uniformly distributed turbulent winds. Journal of Wind Engineering and Industrial Aerodynamics, 2018, 172: 31-41.

[24] C Mannini, A M Marra, T Massai, et al. Interference of vortex-induced vibration and transverse galloping for a rectangular cylinder. Journal of Fluids and Structures, 2016, 66: 403-423.

[25] K Zhang, H Katsuchi, D Zhou, et al. Numerical simulation of vortex induced vibrations of a flexibly mounted wavy cylinder at subcritical Reynolds number. Ocean Engineering, 2017, 133: 170-181.

[26] P Xie, CY Zhou. Numerical investigation on effects of rivulet and cable oscillation of a stayed cable in rain-wind-induced vibration. Journal of Mechanical Science and Technology, 2013, 27(3): 685-701.

[27] H Liu, D Chen, L Hu, et al. Numerical investigations on flow-induced vibration of fuel rods with spacer grids subjected to turbulent flow. Nuclear Engineering and Design, 2017, 325: 68-77.

[28] Y C Su. A study on the flow-induced vibration and heat transfer characteristic of elastic tube bundle. Jinan: Shandong University, 2012. (in Chinese)

[29] D R Duan, P Q Ge, W B Bi, et al. Numerical investigation on the heat transfer enhancement mechanism of planar elastic tube bundle by flowinduced vibration. International Journal of Thermal Sciences, 2017, 112 : 450-459.

[30] Y L Lee, M E Barkey, H T Kang. Metal fatigue analysis handbook. London: Butterworth-Heinemann, 2012.

\section{Submit your manuscript to a SpringerOpen ${ }^{\circ}$ journal and benefit from:}

- Convenient online submission

- Rigorous peer review

- Open access: articles freely available online

- High visibility within the field

Retaining the copyright to your article

Submit your next manuscript at springeropen.com 